\title{
OPINIÓN DE LOS VETERINARIOS DE ATENCIÓN PRIMARIA DE ANDALUCÍA SOBRE LA GESTIÓN DE SU EJERCICIO PROFESIONAL
}

\author{
Francisco Conti Cuesta (1), María Isabel Baena Parejo (2) y Sandra Arlette Pinzón Pulido (3). \\ (1) Distrito Sanitario de Loja-Santa Fe. Granada. \\ (2) Delegación Provincial de Salud. Granada. \\ (3) Escuela Andaluza de Salud Pública. Granada.
}

\section{RESUMEN}

Fundamento: Fste trahajo describe la opinión de los veterinarios de Andalucia sobre la organización y gestión de su ejercicio profesional y se identifican soluciones para los problemas encontrados, con el fin de aportar elementos de planificación y gestión frente a las reformas contempladas en el nuevo Plan Estratégico del Servicio Andaluz de Salud (SAS).

Método: Estudio descriptivo de opinión, utilizando un cuestionario autoadministrado con el total de los veterinarios de Atención Primaria (AP) de Andalucia (incluidos activos con al menos un año de experiencia, excluidos los sustitutos). Se describieron sus caracteristicas personales, su opinión de los factores de gestión de su ejercicio profesional. También se describen las propuestas de mejora a los problemas encontrados.

Resultados: Más del 70\% dicen conocer los objetivos, aunque sólo los coordinadores los consideran claramente motivadores del ejercicio. Piensan que los indicadores de actividad no incluyen criterios de calidad, ni permiten evaluar la actividad que realizan. Creen que las aportaciones de otros miembros del equipo multidisciplinar mejoran su trabajo y apuestan por mantener su actividad en el mismo. Están satisfechos con la realización de su trabajo (en mayor medida los coordinadores). Consideran insuficiente la cantidad de productividad pagada y que ésta no es útil para incentivarlos. No están conformes con la formación que reciben y piensan que su oferta es muy inferior a la de sus compañeros asistenciales. I a mayoría opina que deberían seguir dependiendo del SAS

Conclusiones: Este colectivo prefiere mantener su actividad en la organización actual, aunque mejorando los siguientes aspectos de gestión: oferta de formación, elementos incentivadores del ejercicio e indicadores de actividad.

Palabras clave: Gestión. Ejercicio profesional. Veterinarios. Administración en salud pública. Atención primaria.

Correspondencia:

Francisco Conti Cuesta

Distrito Sanitario Loja-Santa Fe

C/La Paz, 2.

18320 Santa $\mathrm{Fe}$

Granada

Correo electrónico: m-pinzon(a)easp.es

\section{ABSTRACT \\ Opinion of the Primary Care Veterinarians of Andalusia Regarding the Management of their Professional Practice}

Background: This study describes the opinion of the veterinarians in Andalusia regarding the organization and management of their professional practice and pinpoints solutions to the problems found for the purpose of providing planning and management aspects in view of the reforms undertaken in the Andalusian Health Service Strategy Plan.

Method: Descriptive opinion poll employing a questionnaire filled out by each individual among all of the Primary Care (PC) veterinarians in Andalusia (including those who have been currently employed who possess at least one year's experience, except substitutes). A description was provided of their personal traits, their opinion of the management-related factors involved in their professional practice. A description was also provided of the suggestions for improving the problems found to exist.

Results: Over $70 \%$ said they were aware of the objectives, although only the coordinators found them to be clearly motivating the practice. Their opinion was that the indictors of activity neither include any quality-related criteria nor afford the possibility of evaluating the activity they are carrying out. They believed that the contributions from other members of the multidisciplinary team improve their work and are in favor of continuing their involvement therein. They were satisfied with doing their work (the coordinators to a greater degree). They found the productivity pay to be too low and not useful as regards providing them with any incentive. They were not satisfied with the training with which they are provided and thought that what they are offered is far less than their other colleagues in the health care field. Most were of the opinion that they should remain under the authority of the Andalusian Health Service.

Conclusions: This group prefers to continue working for the same organization, but improving the following management aspects: training offer, practice incentives and activity indicators.

Key words: Management. Professional practice. Veterinarians. Public Health Care Management. Primary care. 


\section{INTRODUCCIÓN}

La Reforma Sanitaria de la Atención Primaria (AP) en Andalucía se realizó, a partir de 1984, poniendo especial énfasis en aspectos como la promoción de la salud, la atención a la comunidad, la accesibilidad, la calidad y el equipo multidisciplinar, con el fin de ofrecer a la población un modelo de atención integral, en el que se ofertan, de forma conjunta, servicios asistenciales y de salud pública1. Este modelo de atención contrasta con el de las restantes Comunidades Autónomas, en las que la gestión de los servicios de salud pública se realiza en una red independiente a la dedicada a la atención asistencial.

Los programas y actividades que ejecutan los técnicos del Área de Salud Pública de Andalucía contribuyen al logro de los objetivos marcados en el Plan Andaluz de Salud'. El colectivo de los veterinarios, como parte de esta Área, se integró en el Servicio Andaluz de Salud (SAS) ${ }^{3}$ en 1990 en dos niveles, en el Distrito de AP como coordinadores veterinarios, y en la Zona Básica de Salud (ZBS) como veterinarios de equipo básico, adoptando un modelo de gestión de su ejercicio profesional acorde al de la red de AP en la que quedó incluido ${ }^{4}$. Los objetivos, líneas de actuación y financiación para la red de AP de salud se fijan anualmente entre la Consejería de Salud y el SAS a través del contrato programa, que introduce dirección por objetivos, vincula los costes a la actividad y establece modelos de incentivos 5 .

Sin embargo, la perspectiva que plantea el nuevo Plan Estratégico del SAS ${ }^{6}$ es la reforma de este modelo organizativo, proponiendo la descentralización de la gestión en unidades clínicas y la creación de unidades de salud pública. Esta reforma cambiaría fundamentalmente la organización y gestión del ejercicio profesional del colectivo veterinario en aspectos determinantes, como la fijación de objetivos e incentivos, las necesidades de formación, coordinación y dirección del recurso humano, la gestión de re- cursos materiales, la comunicación interna y el trabajo en equipo, entre otros. Estos cambios, que traen implícita la necesidad de adaptación de la cultura de la organización, podrían ser más eficientemente implantados si, previo a la planificación, se analiza el modelo actúa, desde la perspectiva de sus propios actores $^{7-10}$.

Este estudio se propone conocer la opinión de los veterinarios de AP de Andalucía sobre las características más relevantes de la actual organización y gestión de su ejercicio profesional, con el fin de aportar elementos para la planificación y gestión del nuevo modelo.

Los objetivos de este estudio son: describir la opinión de los veterinarios de Andalucía (coordinadores de Distrito y veterinarios de zona básica de salud), sobre la organización y gestión de su ejercicio profesional, e identificar los elementos de mejora que proponen a los problemas o limitaciones encontrados en la actual organización y gestión de su ejercicio profesional.

\section{SUJETOS Y MÉTODO}

Se realizó un estudio descriptivo transversal ${ }^{11}$ en el ámbito de la red de AP del SAS, durante los meses de julio y agosto de 1998.

La población estudiada fue la formada por los 475 veterinarios de los servicios de AP de Salud de Andalucía, agrupados según el puesto de trabajo que ocupaban: coordinador veterinario de Distrito (50) y veterinario de ZBS (425). Participaron en el estudio los profesionales que cumplían con los criterios de inclusión (estar en activo en la fecha del inicio de la investigación y haber trabajado al menos un año en la red de AP de salud del SAS); se excluyeron los profesionales que hacían las sustituciones de vacaciones.

Para conseguir los objetivos del trabajo se diseñó un cuestionario autoadministrado (de elaboración propia), cstructurado en 58 ítems, que fue remitido por correo a los veterinarios (Anexo 1). En él se recogieron las 
características personales y laborales del sujeto (variables independientes): provincia de origen, edad en años cumplidos (categorizada en $<37$ años, de 37 a 45 años y $>45$ años), género, puesto de trabajo (coordinador o veterinario de ZBS), situación de la ZBS donde trabaja (reconvertida o no reconvertida), relación laboral (funcionario de carrera, funcionario interino o contratado), ejercicio anterior a abril de 1990 y formación de post-grado (ninguna formación, 60 horas y $>60$ horas; en los últimos tres años).

Las preguntas relativas a los aspectos de la organización correspondientes al primer objetivo (48 ítems), se midieron a través de la escala de Likert de 7 puntos, y para su análisis se recodificaron en tres categorías, Desacuerdo (incluye el valor 1, 2 y 3), Indiferencia (incluye el valor 4) y Acuerdo (incluye los valores 5, 6 y 7), a excepción de la variable de comunicación interna, cuyas opciones de respuesta fueron de sí o no. Las dos preguntas para la identificación de elementos de mejora a los problemas o limitaciones encontrados en la organización y gestión de su ejercicio profesional (segundo objetivo) se dejaron abiertas.

Para validar el contenido del cuestionario se utilizaron las siguientes técnicas: 1) identificación de las dimensiones de estudio mediante una revisión bibliográfica de las características más relevantes de las organizaciones ${ }^{2-20}$; 2) consulta a "informantes clave" (profesional veterinario con más de dos años de experiencia en la red de A.P. de salud de
Andalucía, que ha presentado algún trabajo de investigación o con formación en gestión). coordinadores y veterinarios de ZBS; 3) revisión preliminar de la construcción de las preguntas por expertos en diseño de cuestionarios; y 4) pilotaje del cuestionario, donde se valoró duración de la cumplimentación, claridad y pertinencia de las preguntas, exhaustividad y exclusión de las categorías de respuesta $^{21}$. De la validación de contenido se obtuvieron 10 dimensiones de análisis: Objetivos, Trabajo en Equipo, Sistema Retributivo, Dirccción, Coordinación, Comunicación Interna, Recursos, Pertenencia, Carrera Profesional y Adscripción al Distrito. Posteriormente se validó su construcción mediante un análisis factorial de componentes principales, que identificó seis de estas dimensiones, las cuales se tomaron como variables dependientes (tabla 1). Se analizó la consistencia interna de cada una de las dimensiones del cuestionario con el coeficiente Alpha de Cronbach. Fueron eliminados del análisis los items que no superaron los criterios generales de validación o los del análisis factorial (endosamiento, poder discriminante, comunalidades, etc.). El valor de cada dimensión se calculó sumando las puntuaciones de los ítems que la componían, y categorizándola de igual forma (Acuerdo, Indiferencia y Desacuerdo).

Para el análisis estadístico se utilizó el programa SPSS versión 7.5. Se realizó una descripción de la población según sus características personales y laborales, y su opinión sobre los distintos ítems de la organización y

Tabla 1

Dimensiones de análisis de la gestión del ejercicio profesional de los veterinarios de atención primaria de Andalucía

\begin{tabular}{|lll|}
\hline$N .^{\circ}$ & DIMENSIÓN & \multicolumn{1}{c|}{ ITEMS } \\
\hline 1 & Objetivos & $2,3,4,5$ \\
2 & Trabajo en equipo & $7,8,9$ \\
3 & Retribuciones & $13,14,15$ \\
4 & Formación & 17,18 \\
5 & Coordinación & $33,34,35,36$ \\
6 & Comunicación Interna & $38,39,40,41,42,43$ \\
\hline
\end{tabular}


gestión de su ejercicio profesional. Para estudiar la posible asociación entre las características personales y laborales de la población y su opinión sobre las dimensiones de análisis se utilizó el test de la Chi-cuadrado.

Por último, para dar respuesta al segundo objetivo, se realizó un análisis descriptivo de frecuencias de las propuestas formuladas, tanto de forma individual como agrupando las mismas en cada una de las dimensiones estudiadas.

\section{RESULTADOS}

El porcentaje de respuesta general fue del $50,7 \%$ (241); para los coordinadores veterinarios del $76 \%$ (38) y para los VZBS del $47,8 \%$ (203).

Las características personales y laborales de los coordinadores y de los veterinarios de ZBS aparecen en la tabla 2. No hubo diferencias significativas entre los que respon- dieron y los que no respecto a las características de edad, género, relación laboral y zona básica de salud reconvertida o no reconvertida, con valores de $p \geq 0,32$.

\section{Opinión de los veterinarios de Atención Primaria de Andalucía}

El $89,5 \%$ de los coordinadores veterinarios (CV) manifestaron haber establecido en su Distrito Sanitario objetivos propios en el año 1998 , y el $72,4 \%$ de los veterinarios de ZBS (VZBS) manifestaron conocerlos. El $70,6 \%$ de los CV y el $75,2 \%$ de los VZBS creían que las actividades que realizan se ajustan a los objetivos fijados en su Distrito Sanitario, y los CV consideran los objetivos un claro elemento motivador de su trabajo. Tanto los CV (70,3\%) como VZBS $(60,2 \%)$ piensan que los indicadores solicitados desde los Servicios Centrales no permiten evaluar la actividad que realizan, manifestando que los indicadores no incluyen criterios de calidad de las labores realizadas.

Tabla 2

Características personales y laborales de los coordinadores veterinarios y veterinarios de ZBS de Andalucía

\begin{tabular}{|c|c|c|c|c|c|}
\hline \multirow{2}{*}{$\begin{array}{l}\text { PROFESIONAL } \\
\text { VARIABLE }\end{array}$} & & \multicolumn{2}{|c|}{ COORDINADORES } & \multicolumn{2}{|c|}{ VETERINARIOS DE ZBS } \\
\hline & & Frecuencia & Porcentaje & Frecuencia & Porcentaje \\
\hline \multirow{2}{*}{ GÉNERO } & MASCULINO & 27 & 71,1 & 166 & 81,8 \\
\hline & FEMENINO & 11 & 28,9 & 31 & 15,3 \\
\hline \multirow{3}{*}{$\begin{array}{l}\text { ZONA BÁSICA } \\
\text { DE SALUD }\end{array}$} & PERDIDOS & & & 9 & \\
\hline & RECONVERTIDA & & & 182 & 89,7 \\
\hline & NO RECONVERTIDA & & & 18 & 8,9 \\
\hline \multirow{4}{*}{ RELACIÓN LABORAL } & PERDIDOS & & & 3 & 1,5 \\
\hline & FUNCIONARIO & 6 & 15,8 & 25 & 12,3 \\
\hline & INTERINO & 31 & 81,6 & 168 & 82,8 \\
\hline & CONTRATADO & 1 & 2,6 & 6 & 3 \\
\hline \multirow{3}{*}{$\begin{array}{l}\text { EJERCICIO ANTERIOR A } \\
\text { ABRIL DE } 1990\end{array}$} & PERDIDOS & & & 4 & 2 \\
\hline & Sí & 32 & 84,2 & 144 & 70,9 \\
\hline & NO & 6 & 15,8 & 55 & 27,1 \\
\hline \multirow{4}{*}{ EDAD } & PERDIDOS & & & 4 & 2 \\
\hline & $<37$ años & 14 & 36,8 & 85 & 41,9 \\
\hline & De 37 a 45 años & 17 & 44,7 & 83 & 40,9 \\
\hline & $>45$ años & 7 & 18,4 & 35 & 17,2 \\
\hline
\end{tabular}


El 70,3\% de los CV y el 73,5\% de VZBS piensa que los veterinarios de AP deben seguir dependiendo del SAS. También, aunque en proporción inferior, los dos grupos manifestaron acuerdo en integrarse en una estructura jerarquizada dependiente de Dirección General de Salud Pública y $\mathrm{P}(55,6 \%$ de los CV y $46,7 \%$ de los VZBS).

Los dos grupos estudiados opinan que deben mantener su ejercicio profesional en el actual equipo multidisciplinar $(86,8 \%$ de los CV y $73,5 \%$ de los VZBS), e igualmente consideran que su trabajo mejora con las aportaciones de otros miembros del equipo. El $63,2 \%$ de los CV y el $57 \%$ de los VZBS prefieren realizar su trabajo en una estructura no asistencial, formada por profesionales de salud pública.

El $89,2 \%$ de los CV prefiere que los veterinarios de ZBS estuvieran adscritos al Distrito Sanitario, manifestando que esta adscripción conseguiría un reparto más equitativo de la carga de trabajo.

El $65,8 \%$ de los CV y el $60,9 \%$ de los VZBS manifiestan que el pago de la productividad por cumplimiento de objetivos no es útil para incentivarlos y que las cantidades que se pagan son insuficientes para motivar a los profesionales.

El 76,7\% de los VZBS cree que el plan de formación de su Distrito no contribuye a formarlos, siendo un $63,3 \%$ de los CV los que opinan lo mismo. Ambos grupos (78,9\% de los CV y 70,8\% de los VZBS) piensan que la Consejería de Salud no ha aumentado la oferta de formación para los veterinarios en los últimos años, y que la formación ofertada a los mismos es, aún, muy inferior a la que se hace a los profesionales asistenciales.

El $73,7 \%$ de los VZBS consideran inadecuada la coordinación con los veterinarios de la Delegación Provincial.

El $4,2 \%$ de los VZBS manifiesta que la mayor parte de la información sobre el Dis- trito la obtenía del director de su Centro de Salud, y el $72,7 \%$ decía recibirla del CV. El $65,8 \%$ de los coordinadores opina que la mayor parte de la información la recibía del director del Distrito.

A ambos grupos les gustaría recibir y dar información sobre el Distrito en reuniones de equipo. Los CV están interesados en recibir información sobre lo que hacen en otros Distritos de Andalucía, y sobre planes futuros; sin embargo los VZBS están más interesados en temas profesionales y de formación. A los CV les gustaría recibir la información del Director del Distrito y a los VZBS del CV (tablas 3 y 4).

Los CV con edades entre 37 y 45 años opinan estar más en desacuerdo con los objetivos que los mayores de 45 años $(\mathrm{p}=0,03)$; de igual forma opinaban los que tienen más de 60 horas de formación, respecto a los de menor formación $(\mathrm{p}=0,03)$. Los VZBS con edades entre 37 y 45 años están más en desacuerdo con las distintas formas de coordinación existente $(\mathrm{p}=0,005)$ y con la comunicación interna actual $(\mathrm{p}=0,05)$ que los mayores de 45 años. No se encontraron diferencias estadísticamente significativas con respecto a otras características personales o laborales (figuras 1 y 2). Se encontró correlación entre la edad y la relación laboral $(-0,53)$ y la edad y la formación post-grado $(-0,12)$.

\section{Propuestas de mejora a los problemas y limitaciones de la actual organización}

La respuesta que aparece con mayor frecuencia es la de aumentar los recursos materiales y la formación, seguida de la de aumentar la plantilla de veterinarios, solucionar la inestabilidad laboral, unificar criterios de actuación profesional y mejorar la comunicación e información. Por dimensiones, la que aparecía con una frecuencia más alta es la de recursos, seguida de coordinación, formación, situación laboral, comunicación interna y dirección (tabla 5). 
Tabla 3

Opinión de los coordinadores veterinarios y veterinarios de ZBS. de Andalucía sobre la gestión de su ejercicio profesional

\begin{tabular}{|c|c|c|c|c|c|c|}
\hline \multirow{3}{*}{ Item } & \multicolumn{6}{|c|}{ RESPUESTAS TIPO LIKERT RECODIFICADAS } \\
\hline & \multicolumn{2}{|c|}{$\% D E S A C U E R D O$} & \multicolumn{2}{|c|}{$\%$ INDIFERENCIA } & \multicolumn{2}{|c|}{$\% A C U E R D O$} \\
\hline & Coordinadores & $\begin{array}{c}\text { Veterinarios } \\
\angle B S\end{array}$ & Coordinadores & $\begin{array}{c}\text { Veterinarios } \\
Z B S\end{array}$ & Coordinadores & $\begin{array}{c}\text { Veterinarios } \\
\text { ZBS }\end{array}$ \\
\hline 2 & 14.7 & 11 & 14.7 & 13.8 & 70.6 & 75.2 \\
\hline 3 & 23.5 & 33.6 & 5.9 & 15.1 & 70.6 & 51.4 \\
\hline 4 & 70.3 & 60.2 & 13.5 & 15.3 & 16.2 & 24.5 \\
\hline 5 & 89.5 & 76.6 & 2.6 & 9 & 7.9 & 14.4 \\
\hline 6 & 31.6 & 42.6 & 10.5 & 8.2 & 57.9 & 49.2 \\
\hline 7 & 13.2 & 16.2 & 0 & 10.3 & 86.8 & 73.5 \\
\hline 8 & 15.8 & 33.5 & 13.2 & 9.7 & 71.1 & 56.8 \\
\hline 9 & 60.5 & 54.5 & 13.2 & 14.8 & 26.3 & 30.7 \\
\hline 10 & 21.1 & 27 & 15.8 & 16 & 63.2 & 57 \\
\hline 11 & 37.8 & 52 & 29.7 & 19.2 & 32.4 & 28.8 \\
\hline 12 & 15.8 & 31 & 21.1 & 16.8 & 63.2 & 52.3 \\
\hline 13 & 50 & 32.7 & 13.2 & 20.3 & 36.8 & 47 \\
\hline 14 & 65.8 & 60.9 & 5.3 & 11.4 & 28.9 & 27.7 \\
\hline 15 & 84.2 & 89.5 & 10.5 & 5.5 & 5.3 & 5 \\
\hline 16 & 28.9 & 51.3 & 18.4 & 13.7 & 52.6 & 35 \\
\hline 17 & 63.2 & 76.7 & 5.3 & 5.4 & 31.6 & 17.8 \\
\hline 18 & 78.9 & 70.8 & 10.5 & 11.9 & 10.5 & 17.3 \\
\hline 19 & 10.5 & 18.4 & 7.9 & 3 & 81.6 & 78.6 \\
\hline 20 & 50 & 74.3 & 21.1 & 8.4 & 28.9 & 17.3 \\
\hline 21 & 28.9 & 67 & 13.2 & 10 & 57.9 & 23 \\
\hline 22 & 57.9 & 78.7 & 7.9 & 5.9 & 34.2 & 15.3 \\
\hline 23 & 34.2 & 54.8 & 18.4 & 19.6 & 47.4 & 25.6 \\
\hline 24 & 28.9 & 39.2 & 5.3 & 22.2 & 65.8 & 38.7 \\
\hline 25 & 33.3 & 34.2 & 11.1 & 19.1 & 55.6 & 46.7 \\
\hline 26 & 2.6 & 13.9 & 5.3 & 6.9 & 92.1 & 79.2 \\
\hline 27 & 18.9 & 13.5 & 10.8 & 13 & 70.3 & 73.5 \\
\hline 28 & 21.6 & 31.8 & 10.8 & 5.5 & 67.6 & 62.7 \\
\hline 29 & 21.1 & 33.7 & 34.2 & 27 & 44.7 & 39.3 \\
\hline 30 & 10.8 & 44.5 & 2.7 & 23.6 & 86.5 & 31.9 \\
\hline 31 & 8.1 & 32 & 2.7 & 23.7 & 89.2 & 44.3 \\
\hline 32 & 34.2 & 43.3 & 2.6 & 10.8 & 63.2 & 45.8 \\
\hline 33 & 18.4 & 39.8 & 21.1 & 19.9 & 60.5 & 40.3 \\
\hline 34 & - & 24.6 & - & 13.6 & - & 61.8 \\
\hline 35 & 34.2 & 49.7 & 15.8 & 15.1 & 50 & 35.2 \\
\hline 36 & 2.6 & 35 & 7.9 & 15.3 & 89.5 & 49.8 \\
\hline 37 & 51.4 & 73.7 & 13.5 & 14.1 & 35.1 & 12.1 \\
\hline 38 & 34.2 & 57 & 18.4 & 14 & 47.4 & 29 \\
\hline 39 & 26.3 & 38.8 & 39.5 & 25 & 34.2 & 36.2 \\
\hline 40 & 44.7 & 47.7 & 18.4 & 15.2 & 36.8 & 37.1 \\
\hline 41 & 7.9 & 22.8 & 42.1 & 20,7 & 50 & 56.5 \\
\hline 42 & 47.4 & 60.6 & 21.1 & 17.7 & 31.5 & 21.7 \\
\hline 43 & 15.7 & 25.5 & 21.1 & 19.4 & 63.2 & 55.1 \\
\hline
\end{tabular}


Tabla 4

Opinión de los coordinadores veterinarios y veterinarios de ZBS de Andalucía sobre la gestión de su ejercicio profesional

\begin{tabular}{|c|c|c|c|c|}
\hline \multirow{3}{*}{ Item } & \multicolumn{4}{|c|}{ RESPUESTAS Si/No } \\
\hline & \multicolumn{2}{|c|}{ Coordinador } & \multicolumn{2}{|c|}{ Veterinarios $Z B S$} \\
\hline & Sí & No & Si & No \\
\hline 1 & 70.6 & 29.4 & 75.2 & 24.8 \\
\hline $44 \_1$ & 65.8 & - & - & - \\
\hline $44 \_2$ & 26.3 & - & 74.3 & - \\
\hline $44 \_3$ & - & - & 1.5 & - \\
\hline $44 \_4$ & 5.3 & - & 3.5 & - \\
\hline $44 \_5$ & - & - & 19.8 & - \\
\hline 446 & - & - & - & - \\
\hline $44 \_7$ & 2.6 & - & - & - \\
\hline $44 \_8$ & - & - & 1.0 & - \\
\hline $45 \_1$ & 100 & - & 69 & 31 \\
\hline $45 \quad 2$ & - & - & 91.6 & 8.4 \\
\hline $45 \_3$ & 78.9 & 21.1 & 8.9 & 91.1 \\
\hline $45 \_4$ & - & - & 54.7 & 45.3 \\
\hline $45 \_5$ & 57.9 & 42.1 & 19.2 & 80.8 \\
\hline 456 & 5.3 & 94.7 & 1.5 & 98.5 \\
\hline $45 \_7$ & 7.9 & 92.1 & 3.9 & 96.1 \\
\hline 458 & & 100 & & 100 \\
\hline $46 \_1$ & 92.1 & 7.9 & 85.2 & 14.3 \\
\hline $46 \_2$ & 50 & 50 & 53.2 & 46.8 \\
\hline $46 \_3$ & 57.9 & 42.1 & 54.7 & 45.3 \\
\hline $46 \_4$ & 2.6 & 97.4 & 3 & 97 \\
\hline $46 \_5$ & 21.1 & 78.9 & 33 & 67 \\
\hline 466 & & 100 & 1.5 & 98.5 \\
\hline $47 \_1$ & 42.1 & 57.9 & 78.3 & 21.2 \\
\hline $47 \_2$ & 47.4 & 52.6 & 53.2 & 46.8 \\
\hline $47 \_3$ & 65.9 & 34.2 & 30 & 70 \\
\hline $47 \ldots$ & 65.8 & 34.2 & 34 & 66 \\
\hline 47.5 & 52.6 & 47.4 & 68 & 32 \\
\hline 476 & 21.1 & 78.9 & 24.6 & 75.4 \\
\hline 47.7 & & 100 & 1.5 & 98.5 \\
\hline 481 & 13.2 & 86.8 & 16.8 & 83.2 \\
\hline $48 \_2$ & 28.9 & 71.1 & 27.7 & 72.3 \\
\hline $48 \_3$ & 52.6 & 47.4 & 43.1 & 56.9 \\
\hline $48 \_4$ & 76.3 & 23.7 & 75.1 & 24.9 \\
\hline $48 \_5$ & - & - & 49.5 & 50.5 \\
\hline $48 \_6$ & 76.3 & 23.7 & 39.6 & 60.4 \\
\hline $48-7$ & 2.6 & 97.4 & 2.5 & 97.5 \\
\hline $48 \_8$ & & 100 & & 100 \\
\hline
\end{tabular}




\section{Figura 1}

Opinión de coordinadores veterinarios sobre la gestión de su ejercicio profesional según rangos de edad

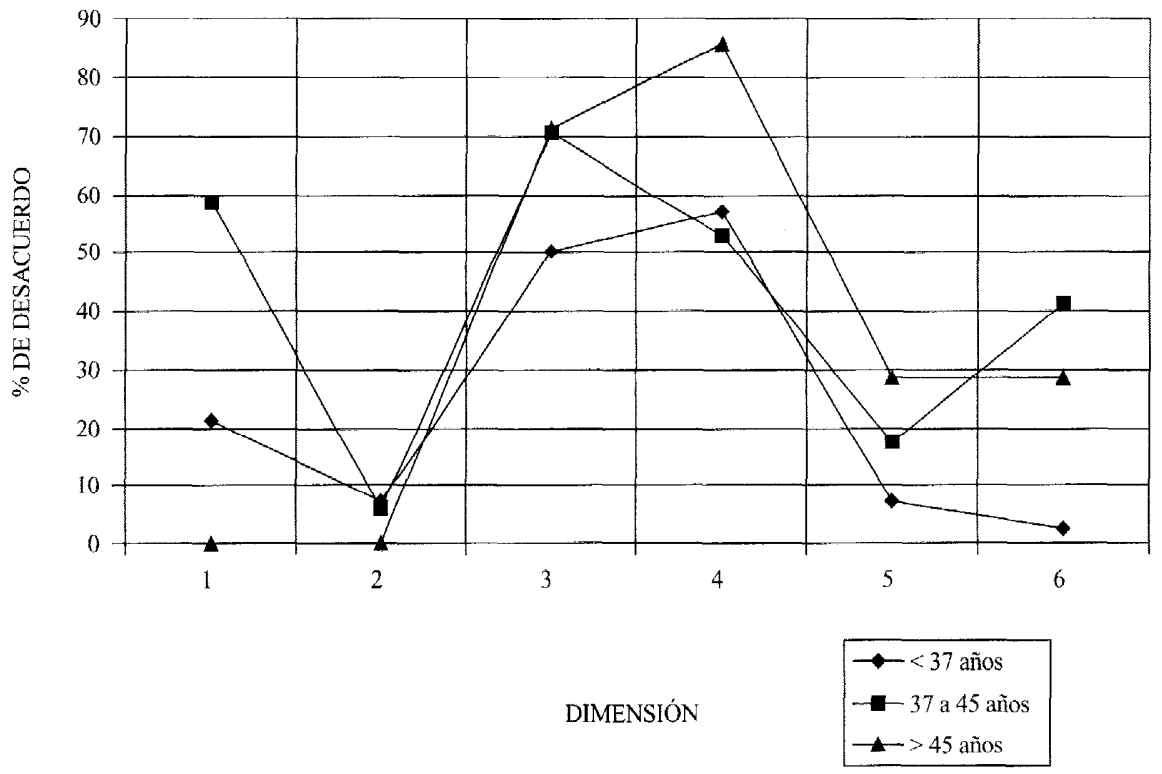

Figura 2

Opinión de veterinarios de ZBS sobre la gestión de su ejercicio profesional según rangos de edad

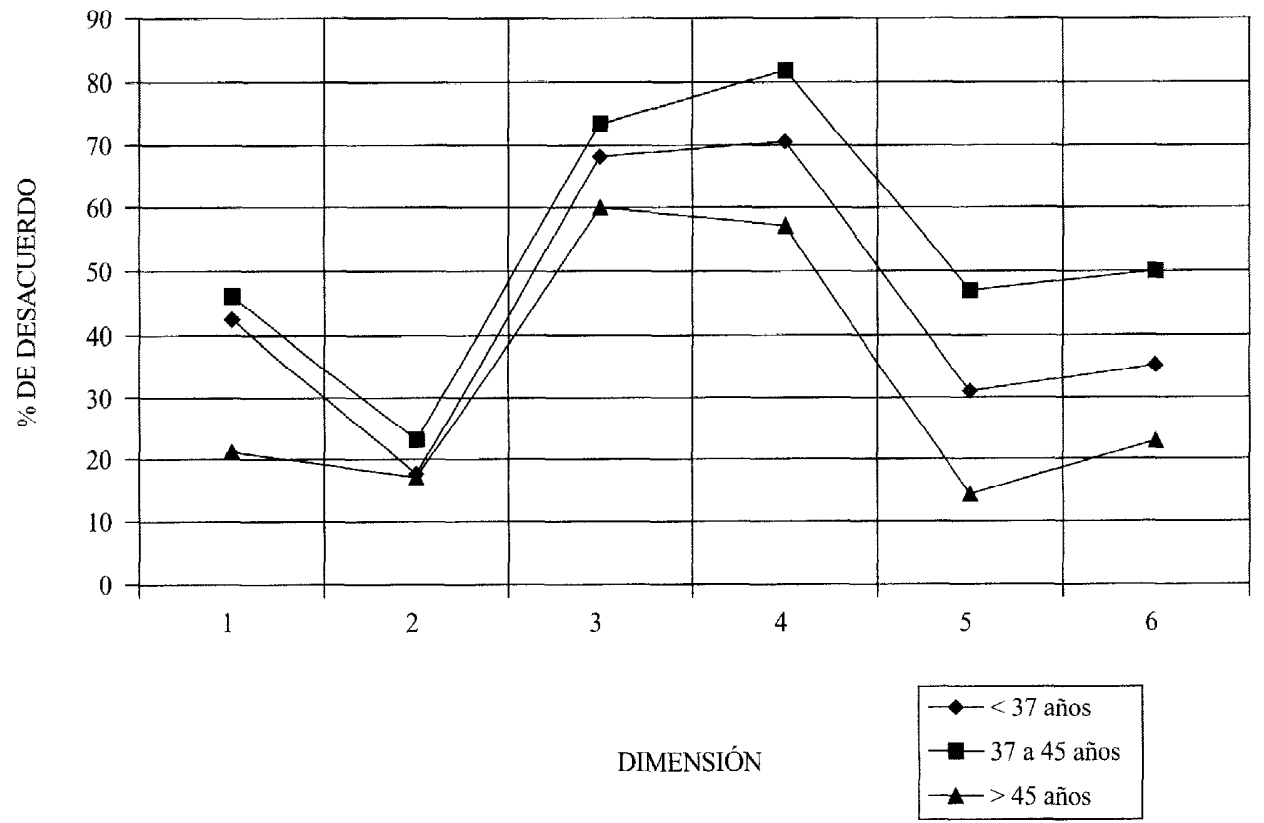

Dimensiones: 1) Objetivos 2) Trabajo en Equipo 3) Retribuciones 4) Formación 5) Coordinación 6) Comunicación Interna.

Fuente: Cuestionarios aplicados a coordinadores y veterinarios de Z.B.S. de Andalucia. Julio 1998 
Tabla 5

Propuestas de mejora de los veterinarios de AP de Andalucía a los problemas o limitaciones en la organización y gestión de su ejercicio profesional

\begin{tabular}{|c|c|c|c|}
\hline DIMENSIÓN & PROBLEMA & FRECUENCIA & PORCENTAJE \\
\hline \multirow{12}{*}{ RECURSOS } & RECURSOS HUMANOS & & \\
\hline & Aumentar la plantilla de veterinarios & 34 & \\
\hline & Dotar de personal administrativo & 14 & \\
\hline & Otros & 6 & \\
\hline & RECURSOS MATERIALES & & \\
\hline & Aumentar recursos materiales & 47 & \\
\hline & Tener coche de la administración & 16 & \\
\hline & Otros & 11 & \\
\hline & RECURSOS ECONÓMICOS & & \\
\hline & Presupuesto específico de S.P. & 12 & \\
\hline & Otros & 12 & \\
\hline & Subtotal & 152 & 32,3 \\
\hline \multirow{3}{*}{ OBJETIVOS } & Mejorar objetivos & 14 & \\
\hline & Otros & 9 & \\
\hline & Subtotal & 23 & 4,9 \\
\hline \multirow{2}{*}{ FORMACIÓN } & Más formación & 45 & \\
\hline & Subtotal & 45 & 9,6 \\
\hline \multirow{3}{*}{ COMUNICACIÓN INTERNA } & Mejor comunicación e información & 20 & \\
\hline & Otros & 7 & \\
\hline & Subtotal & 27 & 5,7 \\
\hline \multirow{2}{*}{ SITUACIÓN LABORAL } & Solucionar la inestabilidad laboral & 33 & \\
\hline & Subtotal & 33 & 7 \\
\hline \multirow{3}{*}{ PERTENENCIA } & Depender de la D.G.S.P. Y P. & 12 & \\
\hline & Otros & 12 & \\
\hline & Subtotal & 24 & 5,1 \\
\hline \multirow{3}{*}{ EQUIPO } & Reconocimiento de la labor & 18 & \\
\hline & Otros & 6 & \\
\hline & Subtotal & 24 & 5,1 \\
\hline \multirow{3}{*}{ COORDINACIÓN } & Unificar criterios de actuación & 21 & \\
\hline & Otros & 36 & \\
\hline & Subtotal & 57 & 12,1 \\
\hline \multirow{3}{*}{ DIRECCIÓN } & Cambiar el coordinador veterinario & 11 & \\
\hline & Otros & 15 & \\
\hline & Subtotal & 26 & 5,5 \\
\hline \multirow{3}{*}{ ADSCRIPCIÓN } & Redistribución plazas veterinarios & 15 & \\
\hline & Otros & 2 & \\
\hline & Subtotal & 17 & 3,6 \\
\hline RETRIBUCIONES & Subtotal & 3 & 0,6 \\
\hline CARRERA PROFESIONAL & Subtotal & 6 & 1,2 \\
\hline . OTROS & Subtotal & 33 & 7 \\
\hline TOTAL & & 470 & 100 \\
\hline
\end{tabular}




\section{DISCUSIÓN}

La inexistencia de un instrumento de medida ya validado obligó a diseñar un cuestionario con tal finalidad. Se eligió una escala de tipo Likert con siete valores, por ser de construcción y respuesta sencilla, aportando también gran cantidad de información.

El porcentaje de respuesta se considera satisfactorio, sobre todo para los CV. Analizando el porcentaje de respuesta de otros estudios realizados en fechas similares y con el mismo tipo de instrumento de recogida de información ${ }^{22-23}$ se comprueba que el porcentaje de respuesta de este trabajo es similar o ligeramente superior.

No hay diferencia significativa entre las características personales y laborales de los veterinarios que respondieron y los que no, por lo que se pueden inferir los resultados obtenidos al total de la población.

En relación a la identificación de pertenencia del colectivo, el seguir adscritos al SAS es claramente la opción mayoritaria pero, por otra parte, a la pregunta sobre su integración en una estructura jerarquizada dependiente de la DGSP y P se obtiene también una respuesta favorable, aunque en menos cuantía que la anterior; el mayor acuerdo hacia la primera opción pudiera estar influida en parte por la resistencia al cambio, atribuible al desconocimiento de la nueva situación, y/o al cambio de estatus por la posibilidad de retribuciones inferiores.

La mayoría del colectivo piensa que debería mantener su ejercicio en el actual equipo multidisciplinar, y un porcentaje algo inferior al anterior prefiere trabajar en un equipo de estructura no asistencial formado por profesionales de salud pública. Estos resultados coinciden con los obtenidos por Luque Lozano ${ }^{24}$, 10 que nos hace pensar que el colectivo no tiene una preferencia exclusiva sobre cual sería la mejor estructura organizativa para el ejercicio de su labor profesional, atribuible, en parte, a la deficiente información que reciben. Sorprende que solo el $4,2 \%$ de los veterinarios de ZBS diga recibir la mayor parte de información del Director del Centro de Salud; este hecho, según el equipo investigador, puede deberse a los problemas de integración del colectivo veterinario en los equipos básicos de AP.

El desacuerdo de los veterinarios de ZBS con su adscripción al distrito puede estar influido por la resistencia al cambio de una medida anunciada desde la empresa, hecho que se podría percibir como la posibilidad de asumir mayor carga de trabajo sin compensación económica alguna. El mayoritario acuerdo sobre este punto de los coordinadores veterinarios indica su idea de mejora en la gestión de los recursos humanos del colectivo.

El haber pasado, en 1998, a la fijación de objetivos por cada Distrito de AP de Andalucía, en lugar de hacerse como hasta ahora desde los servicios centrales a través del Contrato Programa, podría haber dado lugar a la mayor proporción de vetcrinarios que dicen conocerlos, respecto a los resultados obtenidos por Chavernas ${ }^{25}$ un año antes. El desacuerdo de todos los veterinarios de AP con los indicadores de evaluación de actividad fijados desde Servicios Centrales, podría llevar a miembros del colectivo a la desmotivación, al pensar que no se les reconocerá determinado trabajo realizado, ni con qué estándares mínimos de calidad se ha hecho ${ }^{26}$.

Ambos colectivos no creen útil el actual sistema de incentivos, ni adecuadas las cantidades que se abonan. Estos resultados coinciden con los obtenidos por Chavernas ${ }^{25}$, y hacen pensar que la productividad actual no ticne capacidad de motivar a los profesionales para conseguir los objetivos de la organización ${ }^{27}$.

El rol directivo de los coordinadores veterinarios podría influir en el interés de información que manifiestan (saber qué hacen en otros distritos y los planes futuros), en contraste con el interés de los VZBS (por temas 
profesionales y de formación), más propio de un rol de profesional de base.

La coordinación que perciben menos adecuada es la que tienen con los veterinarios de la Delegación Provincial, sobre todo los VZBS. Nos parece que este resultado es la consecuencia de la no pertenencia al mismo organismo (SAS) de dos niveles de la estructura de salud pública.

Las diferencias de opinión encontradas dentro de un mismo colectivo con respecto a la edad pueden tener relación con un perfil determinado del profesional: los profesionales en edades entre 37 y 45 años frecuentemente tienen una relación contractual de tipo de interinidad y suelen ser los que más horas de formación post-grado han recibido. Resulta muy probable encontrar menos acuerdo con la actual organización en este grupo, que entre los mayores de 45 años, entre los que hay más funcionarios que tienen menos horas de formación que los otros.

En las propuestas de mejora a los problemas y limitaciones en la organización y gestión del ejercicio profesional no se han obtenido los resultados deseados ya que, cuando se hace referencia a mejoras en gestión, las respuestas resultan frecuentemente muy generales. Esto se debe, en parte, a que las preguntas realizadas en el cuestionario no permitían delimitar y profundizar en el tema, por lo que creemos más apropiado el abordaje del mismo con una metodología cualitativa.

Se apunta la necesidad de un aumento de plantilla de veterinarios aunque, por otra parte, también se propone una adecuada redistribución de la plantilla existente; por lo que podríamos plantearnos hasta qué punto esta segunda medida podría compensar la primera demanda.

La inestabilidad laboral y la falta de reconocimiento por parte de otros profesionales, figuran entre las propuestas más indicadas a mejorar. Este resultado coincide con las preocupaciones más importantes detectadas en el trabajo realizado por Luque Lozano y $\operatorname{cols}^{24}$.

\section{AGRADECIMIENTOS}

A José Martínez Olmos, por su dirección y lectura critica de la versión previa de este trabajo. A Emilio Perea-Milla López, por su ayuda en el análisis estadístico, y a Ana Conti Beriso por su ánimo y comprensión.

\section{BIBLIOGRAFÍA}

1. Junta de Andalucía. La Reforma Sanitaria en Atención Primaria 1984-1990. Sevilla: Consejería de Salud y Consumo; 1985.

2. Junta de Andalucía. Plan Andaluz de Salud. Sevilla: Consejería de Salud; 1993.

3. Boletín Oficial de la Junta de Andalucía. Ley 8/ 1986 , de 6 de mayo, por la que se crea el Servicio Andaluz de Salud. BOJA núm 41, 10/5/1986.

4. Boletín Oficial de la Junta de Andalucía. Decreto 214/1988, de 17 de mayo, de Reestructuración de los Servicios Oficiales Veterinarios de Andaluć́a. BOJA núm 42, 31/5/1988.

5. Junta de Andalucía. Contrato programa. Sevilla: Consejería de Salud; 1997.

6. Junta de Andalucía. Plan Estratégico del Servicio Andaluz de Salud. Sevilla: Consejería de Salud; 1997.

7. Beckhard, R. Desarrollo organizacional: estrategias y modelos. Madrid: Closas-Orcoyen, SL; 1988.

8. García-Gómez MT. El conflicto y estrategias de negociación. En: Rodríguez-Fernández A, compilador. Los recursos humanos en las Administraciones Públicas. Madrid: Tecnos SA; 1996, p. 439-65.

9. Fernández-Luna, J. Casado, JM. El papel del líder en los procesos de cambio. Capital Humano 1997; (100): 66-70

10. Stoner JA. Freeman R. Administración. México: Prentice Hall; 1994, p. 616-8

11. Bobenrieth M. Burgos, R. Calzas, A. Chicharro, JA. Ocaña R. Perea-Milla E. Sánchez-Cantalejo E. Metodología de investigación y escritura científica en clínica. Granada: Escuela Andaluza de Salud Pública; 1996, p. 31-124 
12. Milgrom P. Roberts, J. Economics, Organization and Management. Nueva Jersey: Prentice Hall. Englewoog-Cliffs; 1992.

13. Gibson JL, Ivancevich JM and Donelly JH. Las organizaciones. Wilmintong, Delaware, USA: Adison-Wesley Iberoamericana SA; 1994.

14. Acámer-Raga F. López-Arribas C. López-Torres-Hidalgo J. Satisfacción laboral de los profesionales sanitarios en Atención Primaria. Aten Primaria 1997; 20 (8): 401-7

15. Rodríguez, R. Márquez, S. López, M. La perspectiva profesional en la reforma de la Atención primaria de Salud: una aproximación cualitativa. Gac Sanit 1995; 49 (9): 237-43

16. Thompson A. Strickland III, AJ. Dirección y administración estratégicas. Conceptos, casos, lecturas. Wilmintong: Addison-Wesley; 1994, p. 2-21

17. Beer M, Spector B, Lawrence P, Mills Q, Walton R. Gestión de recursos humanos. Madrid: Centro de Publicaciones del Ministerio de Trabajo; 1989 , p. 463-506.

18. Thompson, JD. Organization in Action: Social Sciences Bases of Administrative Theory. En: Stoner, JA. Freeman, R. Administración. México: Prentice Hall.: 1994, p. 333-66

19. Villafañe, J. Imagen Positiva: Gestión Estratégica de la Imagen de las Empresas. Madrid: Pirámide; 1998, p. 237-61

20. Rodríguez-Fernández, A, compilador. Los recursos humanos en las Administraciones Públicas. Madrid: Tecnos SA; 1996, p. 37-65
21. Steiner LD, Geoffrey RN. Health measurement scales: a practical guide to their development and use. New York: Oxford University Press; 1989.

22. Ruiz Romero, JA. Actitud de los médicos hacia la política farmacéutica y medidas de uso racional del medicamento en lus Distritos de Alención Primaria (Trabajo de Campo). Granada: Escuela Andaluza de Salud Pública; 1997.

23. Baena Parejo, MI. Estudio de la actitud de los médicos de atención primaria de la provincia de Córdoba hacia la política farmacéutica (Trabajo de Campo). Granada: Escuela Andaluza de Salud Pública; 1992.

24. Luque Lozano, S. De la Torre Molina, R. Torres Medina, JM. Jurado Pérez R. Pérez $\Lambda$ paricio J. Servicios veterinarios y nueva salud pública: situación y valoración por parte de los profesionales de Andalucía. Rev Higea 1997: 3-8.

25. Chavernas, F. Opinión de veterinarios y farmacéuticos de Granada, sobre el contrato-programa (Trabajo de Campo). Granada: Escuela Andaluza de Salud Pública; 1997.

26. Hezberg, F. Una vez más: ¿Cómo motivar a sus empleados? Harvard Business Review 1968: 4-15

27. Miguel-Fernández E. Introducción a la gestión "management": Volumen I. Valencia: Universidad Politécnica de Valencia, 1991, p. 617-63; 675-727. 


\section{Anexo 1}

\section{Cuestionario}

\begin{tabular}{|c|c|c|}
\hline 1 & Se han establecido objetivos en mi Distrito para los profesionales veterinarios en 1998 & Si/No \\
\hline 2 & Las actividades profesionales que realizo se ajustan a estos objetivos & Likert \\
\hline 3 & Conseguir los objetivos del Distrito es un elemento motivador de mi trabajo & Likert \\
\hline 4 & $\begin{array}{l}\text { Los indicadores de actividad solicitados por el SAS y la Delegación permiten evaluar las acti- } \\
\text { vidades desarrolladas por los veterinarios de A.P. }\end{array}$ & Likert \\
\hline 5 & $\begin{array}{l}\text { Los indicadores de actividad solicitados incluyen criterios de calidad de las labores realiza- } \\
\text { das }\end{array}$ & Likert \\
\hline 6 & $\begin{array}{l}\text { Considero que las actividades que realizo son de interés para los profesionales del equipo } \\
\text { multidisciplinar }\end{array}$ & Likert \\
\hline 7 & $\begin{array}{l}\text { Creo importante que los veterinarios mantengamos el ejercicio profesional en el actual equi- } \\
\text { po multidisciplinar }\end{array}$ & Likert \\
\hline 8 & Mi trabajo mejora con las aportaciones de otros miembros del equipo & Liken \\
\hline 9 & $\begin{array}{l}\text { Pienso que trabajando individualmente podría conseguir los mismos resultados en mi ejerci- } \\
\text { cio profesional que haciéndolo en equipo }\end{array}$ & Likert \\
\hline 10 & $\begin{array}{l}\text { Preferiria realizar mi trabajo en una estructura no asistencial formada solo por profesionales } \\
\text { de salud pública (veterinarios, farmacéuticos, etc.) }\end{array}$ & Likert \\
\hline 11 & Me siento más miembro del SAS que miembro de la profesión veterinaria & Likert \\
\hline 12 & Me encuentro satisfecho con la realización de mi trabajo & Likert \\
\hline 13 & Estoy satisfecho con las retribuciones básicas que recibo & Likert \\
\hline 14 & $\begin{array}{l}\text { El pago de productividad por cumplimiento de objetivos es útil para incentivar a los profesio- } \\
\text { nales veterinarios }\end{array}$ & Likert \\
\hline 15 & $\begin{array}{l}\text { Creo que las cantidades que se pagan en concepto de productividad son suficientes para moti- } \\
\text { var a los profesionales sanitarios }\end{array}$ & Likert \\
\hline 16 & En mi Distrito la productividad se asigna de acuerdo a criterios previamente establecidos & Likert \\
\hline 17 & $\begin{array}{l}\text { Creo que el plan de formación de mi Distrito contribuye a la formación de los profesionales } \\
\text { veterinarios }\end{array}$ & Likert \\
\hline 18 & $\begin{array}{l}\text { La formación ofertada a los veterinarios de AP desde la Consejería de Salud se ha incremen- } \\
\text { tado con el tiempo }\end{array}$ & Likert \\
\hline 19 & $\begin{array}{l}\text { Pienso que la formación ofertada a los veterinarios es aún muy inferior a la de otros profesio- } \\
\text { nales asistenciales (médicos y enfermeras) }\end{array}$ & Likert \\
\hline 20 & Dispongo de los recursos materiales necesarios para el ejercicio de mis funciones & Likert \\
\hline 21 & Los veterinarios se sustituyen teniendo en cuenta las necesidades de cada zona & Likert \\
\hline 22 & Dispongo de los recursos de personal auxiliar necesarios para el ejercicio de mis funciones & Liker \\
\hline 23 & $\begin{array}{l}\text { Considero que el Director de Distrito aúna de forma adecuada las actividades de todos los } \\
\text { profesionales sanitarios }\end{array}$ & Likert \\
\hline 24 & El Director valora positivamente el trabajo que realizan de los veterinarios de A.P. & Likert \\
\hline 25 & El colectivo veterinario debería depender de la D.G.S.P. y P. & Likert \\
\hline 26 & $\begin{array}{l}\text { Creo que la figura del coordinador veterinario es necesaria para la organización del trabajo de } \\
\text { los veterinarios de A.P. }\end{array}$ & Likert \\
\hline 27 & Pienso que los veterinarios de A.P. debería seguir dependiendo del SAS & Likert \\
\hline 28 & $\begin{array}{l}\text { Los veterinarios tenemos posibilidades de ocupar puestos de más responsabilidad en la orga- } \\
\text { nización a la que pertenecemos }\end{array}$ & Likert \\
\hline 29 & Me resultan atrayentes los puestos que podría ocupar & Likert \\
\hline 30 & Preferiría estar adscrito al Distrito Sanitario que pertenecer al equipo de Z.B.S. & Likert \\
\hline 31 & $\begin{array}{l}\text { La adscripción de los veterinarios a los Distritos conseguiria un reparto más equitativo de su } \\
\text { carga de trabajo }\end{array}$ & Likeri \\
\hline 32 & Mc pareceria bien asumir funciones asignadas a los veterinarios autorizados & \\
\hline 33 & $\begin{array}{l}\text { Creo que la coordinación que existe para el ejercicio profesional, entre los miembros de mi } \\
\text { equipo es: Adecuada }\end{array}$ & Likert \\
\hline 34 & $\begin{array}{l}\text { Creo que la coordinación que existe para el ejercicio profesional con el coordinador veterina- } \\
\text { rio es: Adecuada }\end{array}$ & Likert \\
\hline 35 & $\begin{array}{l}\text { Creo que la coordinación que existe para el ejercicio profesional con otros miembros del } \\
\text { equipo del Distrito es: Adecuada }\end{array}$ & Likert \\
\hline 36 & Creo que la coordinación que existe con el resto de los veterinarios del Distrito es: Adecuada & Likert \\
\hline
\end{tabular}


37 Creo que la coordinación que existe para el ejercicio profesional con los veterinarios de la Delegación es: Adecuada

Likcrt

38 La información que recibo sobre mi trabajo y sobre el Distrito Sanitario en general es: Abundante

Likert

39 La información que recibo sobre mi trabajo y sobre el Distrito Sanitario en general es: Clara

Likert

40 La información que recibo sobre mi trabajo y sobre el Distrito Sanitario en general es: Periódica

41 La información que recibo sobre mi trabajo y sobre el Distrito Sanitario en general es: Creible

Likert

Likert

42 La información que recibo sobre mi trabajo y sobre el Distrito Sanitario en general es: Llega a tiempo

Likert

43 La información que recibo sobre mi trabajo y sobre el Distrito Sanitario en general es: A través de canales formales

44 La mayor parte de la información que tengo sobre el Distrito la recibo de: 1) Director de Distrito, 2) Coordinador, 3) Otros coordinadores, 4) Director Centro de Salud, 5) Compañeros, 6) Medios de comunicación, 7) Sindicatos, 8) Otros

De quién le gustaría recibir información sobre aspectos generales del Distrito? 1) Director de Distrito, 2) Coordinador, 3) Otros coordinadores, 4) Director Centro de Salud, 5) Compañeros, 6) Medios de comunicación, 7) Sindicatos, 8) Otros

46 A través de qué canales le gustaría recibir información sobre el Distrito 1) Reuniones de equipo, 2) Entrevista individual, 3) Boletines, 4) Tablones, 5) Cartas personales, 6) Otros

47 ¿De qué temas le gustaría recibir información? 1) Formación. 2) Objetivos del Distrito, 3) Planes futuros, 4) Qué se hace en otros Disiritos, 5) Temas profesionales, 6) Avances cientificos, 7) Otros

A través de qué canales le gustaría dar su opinión sobre el Distrito 1) Buzón, 2) Encuesta periódica, 3) Grupos de discusión, 4) Reuniones de equipo, 5) Entrevistas con coordinador, 6) Reuniones con la Dirección, 7) Cartas a la Dirección, 8) Otros Indique los tres problemas o limitaciones que considere más relevantes en la organización y gestión de su ejercicio profesional

50 Indique las propuestas para mejorar los tres problemas o limitaciones que considere más relevantes en la organización y gestión de su ejercicio profesional

Likert

Categorias

Una respuesta

$$
\text { Sí/No }
$$

Opción múltiple

Si/No

Opción múltiple

Síno

Opción múltiple

Síno

Opción múltiple

Abierta

Provincia de Origen

Abierta

Categorías

Edad

Continua

Género

Dicotómica

Puesto de trabajo

Categorías

Situación de la Z.B.S. donde trabaja

Categorías

Relación laboral

Categorías

Ejercicio anterior a Abril de 1990

$\mathrm{Si} / \mathrm{No}$

$58 \quad$ Formación de post-grado

Categorías 